\title{
Poor dietary intake improved by total excision of oral cavity metastases in a patient with hepatocellular carcinoma and elevated myeloid-derived suppressor cells
}

\author{
Ching Chang', Chien-Hao Huang ${ }^{2,3}$ \\ ${ }^{1}$ Department of Internal Medicine, Chang-Gung Memorial Hospital, Taoyuan; ${ }^{2}$ Division of Hepatology, Department of Gastroenterology and \\ Hepatology, Chang-Gung Memorial Hospital, Linkou Medical Center, Taoyuan; ${ }^{3}$ Chang-Gung University, College of Medicine, Taoyuan \\ Correspondence to: Chien-Hao Huang, MD, PhD. Division of Hepatology, Department of Gastroenterology and Hepatology, Chang-Gung Memorial \\ Hospital, Linkou Medical Center, Taiwan Chang-Gung University, College of Medicine, Taoyuan. Email: huangchianhou@gmail.com. \\ Provenance and Peer Review: This article was not commissioned by the editorial office. The article was sent for external peer review.
}

Submitted Dec 16, 2019. Accepted for publication Mar 13, 2020.

doi: $10.21037 /$ hbsn.2020.03.21

View this article at: http://dx.doi.org/10.21037/hbsn.2020.03.21

Hepatocellular carcinoma (HCC) is a common form of cancer, and it ranked sixth for cancer incidence and fourth for cancer deaths globally in 2015 (1). HCC treatment strategies include resection, transplantation, ablation, local therapy, systemic therapy, and palliative care depending on the stage, where metastasis indicating poor prognosis (2). Rare HCC metastases such as those in the oral cavity indicate aggressive malignancy and even poorer outcome, and it may be the initial manifestation of HCC (2).

Oral metastatic neoplasms are seldom observed in practice, and usually involve the mandible when they arise. HCC and adenocarcinoma are the major histopathological findings (3). Even more rare are reports of improved dietary intake following tumor excision. Myeloid-derived suppressor cells (MDSC) strongly increase during cancer, chronic infection, and other illnesses, and are associated with the onset of HCC (4) as well as metastasis (5). For these reasons, our experience with a case involving advanced HCC and left superior gingival metastasis with high blood MDSC offers a unique learning opportunity. Notably, the patient's appetite and nutrition status both improved following total tumor excision.

A 53-year-old male with hepatitis B-related liver cirrhosis was diagnosed with HCC upon initial complaint of epigastric fullness. The cancer was staged at T3bN0M0, with two main tumors $(10 \mathrm{~cm}$ over Segment 6/7, $7 \mathrm{~cm}$ over Segment 8 ) and portal vein, superior mesenteric vein, and splenic vein thrombosis. After histological confirmation, combined hepatic arterial infusion chemotherapy (HAIC) and proton therapy were started.

However, after eight cycles of a cisplatin and 5-fluorouracil HAIC regimen, the disease progressed with multiple intrahepatic tumor recurrences-the largest $5.6 \mathrm{~cm}$ in length. At the same time, a rapidly growing mass over the patient's left superior gingival line over the span of 2 weeks was noted (Figure 1A). 18F-FDG positron-emission tomography (PET) images were also consistent (Figure 1B). This mass caused oral pain and poor dietary intake. Concurrently, granulocytic MDSC among peripheral blood mononuclear cells showed were measured at $40.30 \%$ (Figure 1C).

Therapy for left superior gingival mass began with radiation therapy after biopsy confirmed metastatic HCC (Figure 1D). However, poor response to treatment led our team to perform a total tumor excision via maxillofacial surgery (Figure 1E). Oral pain and poor dietary intake both greatly improved following surgery. Post-surgery nutritional parameters such as body weight gained from 60.6 to $61.6 \mathrm{~kg}$, serum albumin improved from 2.24 to $2.44 \mathrm{~g} / \mathrm{dL}$, and serum creatine also improved from 0.36 to $1.12 \mathrm{mg} / \mathrm{dL}$. Meanwhile, liver function tests such as alanine transaminase (ALT) decreased from 103 to $77 \mathrm{U} / \mathrm{L}$, and prothrombin time shortened from 17.5 to 16.8 seconds. However, due to tumor progression and rupture, total bilirubin deteriorated slightly from 3.3 to $4.3 \mathrm{mg} / \mathrm{dL}$. The patient died the following month due to liver tumor rupture.

Common extra-hepatic metastasis sites for HCC include the lungs, intra-abdominal lymph nodes, adrenal glands, and bone (vertebrae, ribs, and long bones) (6). Oral cavity 

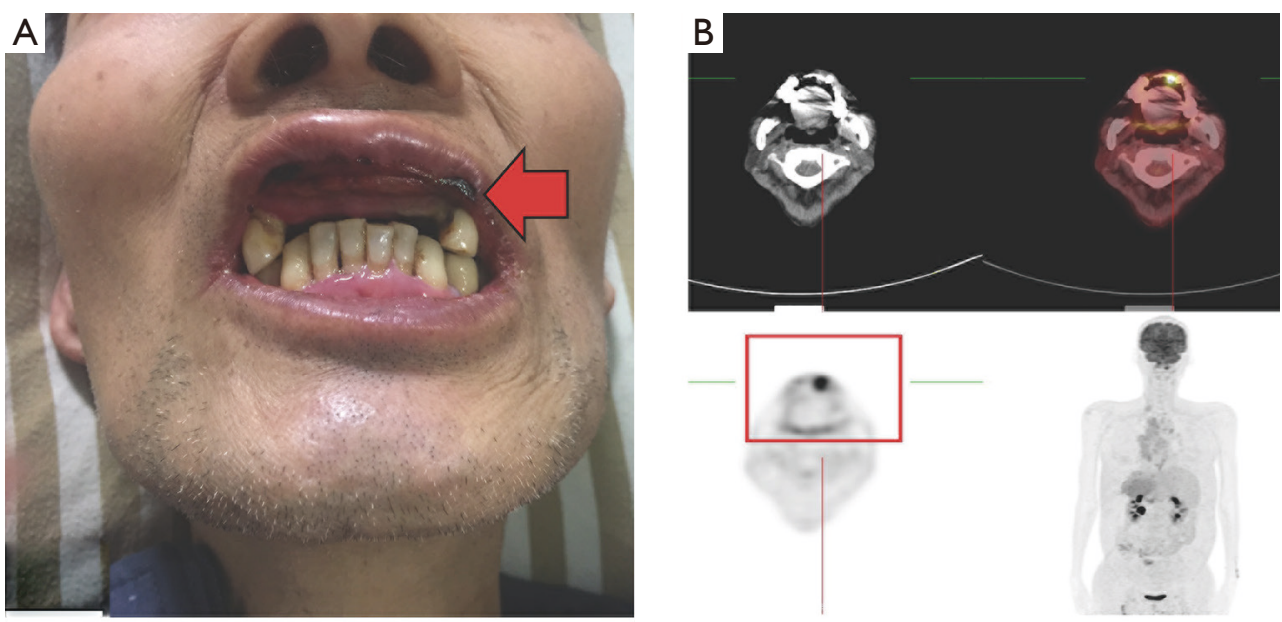

C

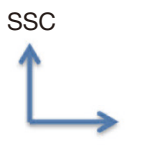

CD45
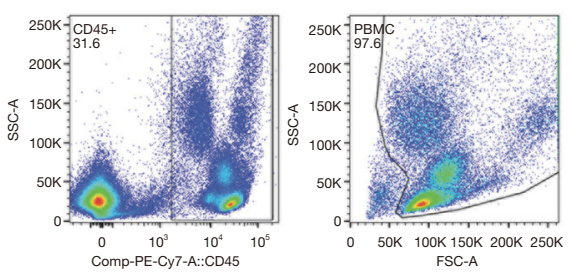

SSC

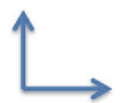

FSC

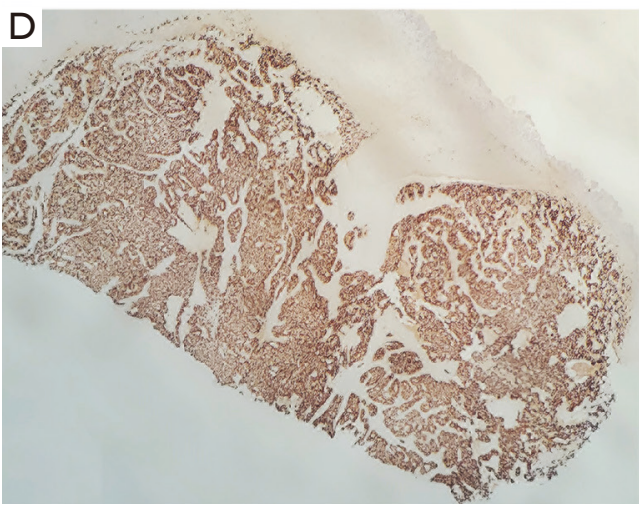

CD11b

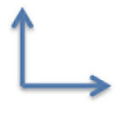

CD33
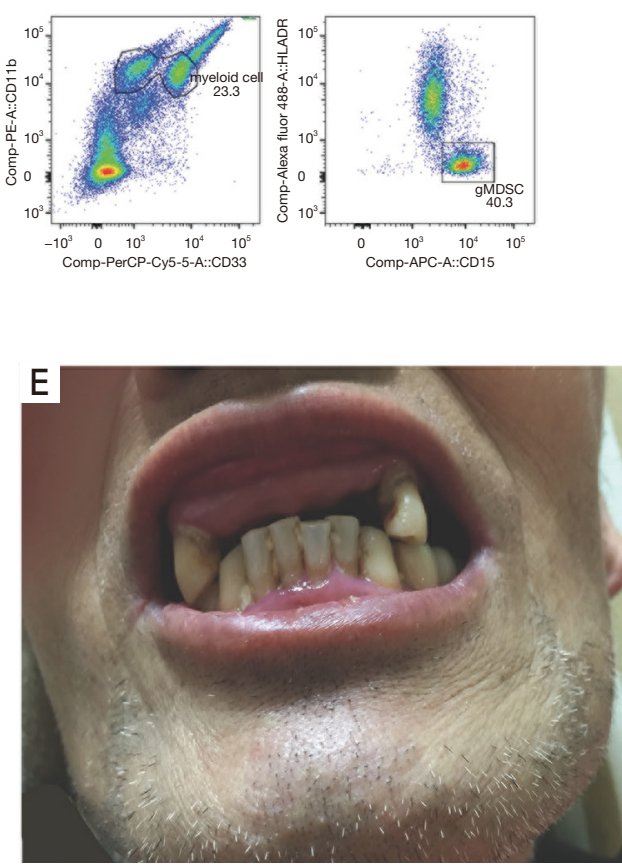

Figure 1 Oral cavity metastases in a patient with HCC and elevated MDSC. (A) A rapidly growing mass over the patient's left superior gingival line over the span of 2 weeks was noted. The red arrow indicates the tumor location. (B) 18F-FDG positron-emission tomography (PET) images were also consistent with the oral cavity metastasis. The black dot in the red box indicates a hypermetabolic mass which is identical to the tumor in (A). (C) Concurrently, granulocytic MDSC among peripheral blood mononuclear cells showed were measured at 40.30\%. (D) Pathology of the left superior gingival mass confirmed metastatic HCC. The immunohistochemical staining showed the tumor cells were positive for Hep-par 1 (original magnification $\times 40$ ). (E) The patient status post total tumor excision via maxillofacial surgery. Oral pain and poor dietary intake both greatly improved following the surgery. HCC, hepatocellular carcinoma; MDSC, myeloid-derived suppressor cell. 
metastasis is rare (2). The most commonly affected oral site for HCC oral metastasis is posterior mandible region, followed by gingiva and parotid gland (7). Jiang et al. used 18F-FDG PET imaging to assess a possible HCC metastasis inside the oral cavity (8). The images showed that the oral lesion was hypermetabolic, which corresponds to our PET image finding.

Major symptoms of metastatic gingiva tumors include pain, dysphagia, and bleeding (2). Maintenance of oral function by tumor removal was mentioned in a study (9), which was important for our patient to obtain better nutrition. In this case, pain from the tumor severely hindered the patient's appetite, leading to a decrease in caloric intake. These discomforts were relieved by excision of the tumor, suggesting that early removal of gingival metastases can correct the loss of appetite and improve nutrition.

HCC with gingival metastasis usually leads to poor prognosis (2), so early discovery and prompt actions are important. In this case, the left superior gingival metastatic tumor was accompanied by the emergence of new liver tumors, ultimately leading to fatal liver rupture one month later. On the other hand, for HCC, MDSCs are reported to play a role in tumor development by altering immune response (4).

MDSCs are distinct from mature myeloid cells (macrophages, dendritic cells, neutrophils) and are active in diseases such as cancer, chronic infection, and autoimmune disease. These cells have also been associated with tumor progression and the presence of solid, metastatic tumors (10).

In this case of advanced HCC with distant metastasis, granulocytic MDSC present among peripheral blood mononuclear cells was as high as $40.30 \%$. This high proportion of MDSC may be related to the tumor progression, distant metastasis, and poor outcome.

\section{Acknowledgments}

The authors thank to all doctors who had taken care of the patient.

Funding: The authors thank NMRPG3G0211 and NMRPG3H6041 for financial support.

\section{Footnote}

Conflicts of Interest: Both authors have completed the ICMJE uniform disclosure form (available at http://dx.doi. org/10.21037/hbsn.2020.03.21). The authors have no conflicts of interest to declare.

Ethical Statement: The authors are accountable for all aspects of the work in ensuring that questions related to the accuracy or integrity of any part of the work are appropriately investigated and resolved. Written informed consent was obtained from the patient for publication of this manuscript and any accompanying images.

Open Access Statement: This is an Open Access article distributed in accordance with the Creative Commons Attribution-NonCommercial-NoDerivs 4.0 International License (CC BY-NC-ND 4.0), which permits the noncommercial replication and distribution of the article with the strict proviso that no changes or edits are made and the original work is properly cited (including links to both the formal publication through the relevant DOI and the license). See: https://creativecommons.org/licenses/by-nc-nd/4.0/.

\section{References}

1. Global Burden of Disease Cancer Collaboration, Fitzmaurice C, Allen C, et al. Global, Regional, and National Cancer Incidence, Mortality, Years of Life Lost, Years Lived With Disability, and Disability-Adjusted Lifeyears for 32 Cancer Groups, 1990 to 2015: A Systematic Analysis for the Global Burden of Disease Study. JAMA Oncol 2017;3:524-48.

2. Pesis M, Taicher S, Greenberg G, et al. Metastasis to the jaws as a first manifestation of hepatocellular carcinoma: report of a case and analysis of 41 cases. J Craniomaxillofac Surg 2014;42:1997-2001.

3. Lee YH, Lee JI. Metastatic carcinoma of the oral region: An analysis of 21 cases. Med Oral Patol Oral Cir Bucal 2017;22:e359-65.

4. Li X, Xing YF, Lei AH, et al. Neutrophil count is associated with myeloid derived suppressor cell level and presents prognostic value of for hepatocellular carcinoma patients. Oncotarget 2017;8:24380-8.

5. Lu C, Rong D, Zhang B, et al. Current perspectives on the immunosuppressive tumor microenvironment in hepatocellular carcinoma: challenges and opportunities. Mol Cancer 2019;18:130.

6. Katyal S, Oliver JH 3rd, Peterson MS, et al. Extrahepatic metastases of hepatocellular carcinoma. Radiology 2000;216:698-703.

7. Rodrigues M, Esteves CM, de Oliveira MA, et al. Fatal hepatocellular carcinoma presenting with oral metastasis in 
a patient with synchronic primary malignancies of prostate and liver. Gerodontology 2017;34:272-5.

8. Jiang L, Gu T, Liu F, et al. A Case of Oral Metastasis From Hepatocellular Carcinoma Displayed on 18F-FDG PET/CT Imaging. Clin Nucl Med 2016;41:72-3.

9. Friedrich RE, Zustin J. Multiple distant metastases of hepatocellular carcinoma to the oral cavity. In Vivo
2010;24:211-4.

10. Diaz-Montero CM, Salem ML, Nishimura MI, et al. Increased circulating myeloid-derived suppressor cells correlate with clinical cancer stage, metastatic tumor burden, and doxorubicin-cyclophosphamide chemotherapy. Cancer Immunol Immunother 2009;58:49-59.

Cite this article as: Chang $\mathrm{C}$, Huang $\mathrm{CH}$. Poor dietary intake improved by total excision of oral cavity metastases in a patient with hepatocellular carcinoma and elevated myeloid-derived suppressor cells. HepatoBiliary Surg Nutr 2020;9(4):558-561. doi: $10.21037 / \mathrm{hbsn} .2020 .03 .21$ 